\title{
Design and performance of a three-wavelength LED-based total scatter and backscatter integrating nephelometer
}

\author{
T. Müller ${ }^{1}$, M. Laborde ${ }^{2,3}$, G. Kassell ${ }^{2}$, and A. Wiedensohler ${ }^{1}$ \\ ${ }^{1}$ Leibniz Institute for Tropospheric Research, Leipzig, Germany \\ ${ }^{2}$ Ecotech Pty Ltd, Knoxfield, Australia \\ ${ }^{3}$ Paul Scherrer Institut, Villigen, Switzerland
}

Received: 5 August 2010 - Published in Atmos. Meas. Tech. Discuss.: 9 November 2010

Revised: 19 April 2011 - Accepted: 7 May 2011 - Published: 29 June 2011

\begin{abstract}
Integrating nephelometers are instruments that directly measure a value close to the light scattering coefficient of airborne particles. Different models of nephelometers have been used for decades for monitoring and research applications. Now, a series of nephelometers (Ecotech models M9003, Aurora 1000 and Aurora 3000) with newly designed light sources based on light emitting diodes are available. This article reports on the design of these integrating nephelometers and a comparison of the Aurora 3000 to another commercial instrument (TSI model 3563) that uses an incandescent lamp. Both instruments are three-wavelength, total and backscatter integrating nephelometers.

We present a characterization of the new light source design of the Aurora 3000 and provide parameterizations for its angular sensitivity functions. These parameterizations facilitate to correct for measurement artefacts using Mie-theory. Furthermore, correction factors are provided as a function of the Ångström exponent. Comparison measurements against the TSI 3563 with laboratory generated white particles and ambient air are also shown and discussed. Both instruments agree well within the calibration uncertainties and detection limit for total scattering with differences less than $5 \%$. Differences for backscattering are higher by up to $11 \%$. Highest differences were found for the longest wavelengths, where the signal to noise ratio is lowest. Differences at the blue and green wavelengths are less than $4 \%$ and $3 \%$, respectively, for both total and backscattering.
\end{abstract}

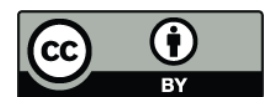

Correspondence to: T. Müller (thomas.mueller@tropos.de)

\section{Introduction}

Integrating nephelometers, first introduced by Beuttell and Brewer (1949), offer a method of measuring the light scattering coefficient of airborne particles. Because no assumptions about particle composition, size or shape are necessary, the use of integrating nephelometers is widespread for ambient air monitoring as well as for laboratory studies. A scientific review of this type of instrument was given by Heintzenberg and Charlson (1996). Integrating nephelometers can be calibrated with gases of well known scattering coefficients, which facilitates quality assurance and maintenance.

Radiative transfer calculations of the Earth's atmosphere require the scattering phase function or at least the asymmetry parameter of airborne particles. One method of estimating the asymmetry parameter is to convert the measured back-scatter fraction, the ratio of hemispheric backscattering to total scattering, into their corresponding asymmetry parameters. Integrating nephelometry for measuring hemispheric backscattering is described in Charlson et al. (1974) and Heintzenberg (1978), and relationships between backscatter fraction and particle asymmetry parameter were investigated by Marshall et al. (1995) and Andrews et al. (2006).

Several studies on the performance of integrating nephelometers (Anderson et al., 1996; Heintzenberg et al., 2006, Müller et al., 2009, Massoli et al., 2009) were performed. Previous intercomparisons of instruments of different makes are limited to total scattering only. We present the first intercomparison of two integrating total/backscatter nephelometers. Additionally, to the backscatter option both nephelometers are three wavelength instruments. One instrument type TSI 3563 (TSI Inc, St. Paul, MN, USA) was characterized in detail by Anderson et al. (1996). In the recent years,

Published by Copernicus Publications on behalf of the European Geosciences Union. 
nephelometers (models M9003, Aurora 1000, and Aurora 3000) with light emitting diodes were developed by Ecotech Pty, Ltd, Knoxfield, Australia.

We present the history of development of the Ecotech Aurora 3000 integrating nephelometer and a comparison of this three wavelength total and backscattering instrument against the TSI model 3563. We discuss the theory of integrating nephelometers and describe the Aurora 3000 instrument. Furthermore, we investigate the characteristic of the angular sensitivity of the new LED light source and provide values to parameterize it. Finally, intercomparison experiments between the two integrating nephelometer types are presented and discussed using ammonium sulfate and ambient aerosols.

\section{Principle of operation of integrating nephelometers and instrument descriptions}

\subsection{Theory of integrating nephelometers}

The total scattering signal $S_{t s, \lambda}$ and hemispheric backscattering signal $\mathrm{S}_{\mathrm{bs}, \lambda}$ measured by integrating nephelometers at wavelength $\lambda$ can be written as

$S_{\mathrm{ts}, \lambda}=\int_{0^{\circ}}^{180^{\circ}} F_{\lambda}(\theta) Z_{\mathrm{ts}}(\theta) d \theta$

and

$S_{\mathrm{bs}, \lambda}=\int_{0^{\circ}}^{180^{\circ}} F_{\lambda}(\theta) Z_{\mathrm{bs}}(\theta) d \theta$,

where $Z_{\mathrm{ts}}(\theta)$ and $Z_{\mathrm{bs}}(\theta)$ are the angular sensitivity functions of the nephelometer for total scattering and backscattering, respectively, and $\theta$ is the scattering angle.

$F_{\lambda}(\theta)=\int_{-\infty}^{+\infty} f_{\lambda}\left(\theta, m_{\lambda}, d_{\mathrm{p}}\right) \frac{d n\left(d_{\mathrm{p}}\right)}{d \log d_{\mathrm{p}}} \pi\left(\frac{d_{\mathrm{p}}}{2}\right)^{2} d \log d_{\mathrm{p}}+F_{\mathrm{R}}(\theta)$

is the angular scattering function of the particle population and the carrier gas. The particle number size distribution is given by $d n\left(d_{\mathrm{p}}\right) / d \log d_{\mathrm{p}}$ and $f_{\lambda}\left(\theta, m_{\lambda}, d_{\mathrm{p}}\right)$ is the angular scattering function of the individual particles with complex refractive $m_{\lambda}$ and diameter $d_{\mathrm{p}} . f_{\lambda}$ can be calculated using light scattering codes. For calculations throughout the manuscript, a code given in Bohren and Huffman (1983) was used. $F_{\mathrm{R}}(\theta)$ is the contribution of the Rayleigh scattering of the carrier gas to the scattering function. Calibration of integrating nephelometers with two particle free gases of known Rayleigh scattering coefficients yields calibration constants $K$ which relate the scattering signals $S$ to the total scattering (subscript ts) and backscattering (subscript bs) coefficients $\sigma$ by

$\sigma_{\mathrm{ts}, \lambda}=K_{\mathrm{ts}, \lambda} S_{\mathrm{ts}, \lambda}-\sigma_{\mathrm{tsR}, \lambda}$ and

$\sigma_{\mathrm{bs}, \lambda}=K_{\mathrm{bs}, \lambda} S_{\mathrm{bs}, \lambda}-\sigma_{\mathrm{bsR}, \lambda}$

where the contribution for Rayleigh scattering $\sigma_{\mathrm{tsR}, \lambda}$ and $\sigma_{\mathrm{bsR}, \lambda}$ are calculated analytically (e.g. Buchholz, 1995) for given temperature and pressure and subtracted from the scattering signals.

Angular sensitivity functions of an ideal nephelometer would be

$Z_{\text {ts }}(\theta)=\sin (\theta)$

for total scattering and

$Z_{\mathrm{bs}}(\theta)=\left\{\begin{array}{cc}\sin (\theta) & 90^{\circ}<\theta<180^{\circ} \\ 0 & \text { otherwise }\end{array}\right.$

for backscattering. The calculated coefficients (Eqs. 1 to 7) are denoted as true total scattering coefficient $\sigma_{\mathrm{ts}}^{\text {true }}$ and true backscattering coefficient $\sigma_{\mathrm{bs}}^{\text {true }}$ for ideal angular sensitivity functions. The angular sensitivity functions of nephelometers are formed by a light source with Lambertian radiant emission meaning that the angular illumination of the particles is almost a sine-function. In the backscattering mode, a shutter prevents forward emitted light from reaching the sample volume. Because illumination functions of integrating nephelometers deviate little from a sine-function (Anderson et al., 1996; Müller et al., 2009), and due to a limited range of angular integration (e.g. Anderson et al., 1996; Moosmüller and Arnott, 2003), integrating nephelometers measure the so called nephelometer total scattering $\sigma_{\mathrm{ts}}^{\text {neph }}$ and nephelometer backscattering $\sigma_{\mathrm{bs}}^{\text {neph }}$. In the following, the term angular sensitivity comprises both the non-Lambertian illumination and the angular truncation. We follow the definition of correction factors from Anderson and Ogren (1998), where the ratios of true to measured nephelometer values for both total scattering and backscattering are defined by

$C_{\mathrm{ts}, \lambda}=\frac{\sigma_{\mathrm{ts}, \lambda}^{\mathrm{true}}}{\sigma_{\mathrm{ts}, \lambda}^{\text {neph }}} \frac{\sigma_{\mathrm{tsR}, \lambda}^{\text {neph }}}{\sigma_{\mathrm{tsR}, \lambda}^{\text {true }}}$

and

$C_{\mathrm{bs}, \lambda}=\frac{\sigma_{\mathrm{bs}, \lambda}^{\text {true }}}{\sigma_{\mathrm{bs}, \lambda}^{\text {neph }}} \frac{\sigma_{\mathrm{bsR}, \lambda}{ }^{\text {neph }}}{\sigma_{\mathrm{bsR}, \lambda}^{\text {true }}}$,

respectively. The second quotients in the above equations compensate for the non-ideal illumination function, when calibrating the nephelometer with Rayleigh scattering gases. In Anderson and Ogren (1998) the compensations for gas calibration is implicitly stated by "To calculate $C$, it is necessary to take the gas calibration ... into account". However, in Eqs. (8) and (9) the full equations are explicitly given.

The correction factors $C_{\mathrm{ts}, \lambda}$ and $C_{\mathrm{bs}, \lambda}$ depend on both particle size and refractive index. If angular sensitivity functions $Z_{\mathrm{ts}}(\theta)$ and $Z_{\mathrm{bs}}(\theta)$ are known, correction factors can be simulated for given particle number size distributions and refractive indices. Easy to use parameterizations for sensitivity functions are given in Sect. 3. 


\subsection{Instrument descriptions}

\subsubsection{Development of the Ecotech model Aurora 3000 integrating nephelometer}

In 2002, the first LED (light emitting diode) based nephelometer with type designation M9003 was developed by Ecotech. The unique feature of this nephelometer was an array of seven LEDs, where the LEDs were shining to the center point of a diffusing plate. The electrical drive current of each LED was adjusted so that the angular intensity distribution of the light source was nearly a Lambertian radiation distribution. The M9003 nephelometer was a single wavelength total scatter only nephelometer. The nephelometer was available at wavelengths of 470,525 , or $630 \mathrm{~nm}$. The performance of the M9003 with wavelength $525 \mathrm{~nm}$ was investigated during nephelometer intercomparisons and calibration workshops (Heintzenberg et al., 2006; Müller et al., 2009). An improvement of the performance of the illumination function was achieved by using 15 LEDs and released as model Aurora 1000. A new three-wavelength light source (used in the original Aurora 3000 model nephelometers) was designed, which included 3 light source boards in one housing with a total of 45 LEDs. In all cases, LEDs of different colours are sequentially switched on and off to allow the detection of scattering signals at all three wavelengths with a single photomultiplier tube. A recalibration of the brightness of each LED in models M9003, Aurora 1000, and Aurora 3000 was time-consuming and could not be done by the user. It is known that an optically thicker opal glass diffuser helps to form a Lambertian illumination function. However, an opal glass reduces the transmitted intensity. With rapid improvements in LED technology, it has become possible to obtain higher intensity outputs from smaller LED packages. Ecotech released an opal glass LED light source in November 2008. This light source utilizes an opal glass diffuser fitted in front of a small array of only 3 high intensity LEDs per wavelength to form a Lambertian distribution. The light source is attached to the sensing volume. The angular integration of nephelometers is limited by apertures at both ends of the sensing volume. For the Aurora 3000 and 1000, the angular integration ranges from $10^{\circ}$ to $171^{\circ}$.

A further development of the Aurora 3000 was to implement a hemispheric backscatter mode. A motor driven backscatter shutter, which is attached to the light source block, moves periodically between two pre-programmed positions. Sketches of the nephelometer cell, light source, and backscatter shutter are given in Fig. 1. The position of the shutter is controlled by a servomotor with position feedback, a servo controller (mounted on the outside of the light source) and a microprocessor. In the backscatter position, the shutter blocks the light emitted in forward direction. The remaining scattered light is seen by the detector as hemispheric backscattering. a)

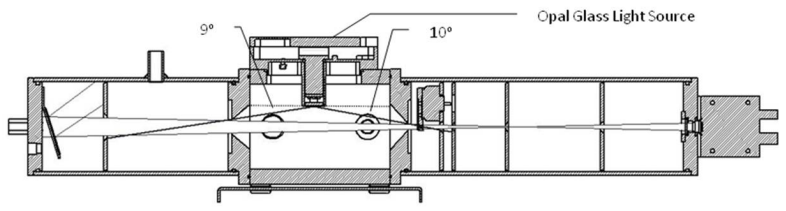

b)

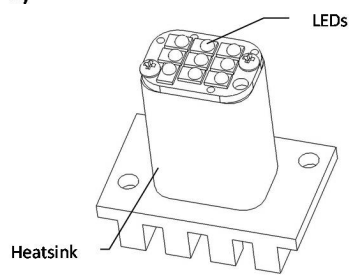

c)

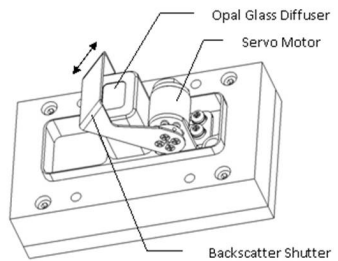

Fig. 1. Sketch of the Aurora 3000. (a) Sensing volume and indicated truncation angles, (b) arrangement of LEDs, (c) light source with motor driven backscatter shutter.

Specifications of the Aurora 3000 are summarized in Table 1.

\subsubsection{TSI model 3563}

For comparison purposes, the integrating nephelometer model TSI 3563 was also used in this study. For a detailed description of the TSI 3563, we refer to Anderson et al. (1996). We briefly summarize the main parameters of interest. The light source of the model 3563 is an incandescent quartz-halogen lamp, which emits light in a large range of wavelengths. An elliptical mirror focuses light onto one end of an optical pipe. The other end of the pipe is attached to an opal glass diffuser providing a nearly Lambertian emission. In the forward scattering direction, an aperture limits the angular integration to angles greater than $7^{\circ}$. At the other side, a shadow plate limits the integration to angles less than $170^{\circ}$. Light scattered by particles enters the detection unit and is split by dichroic filters into three light beams of separated spectral ranges. Interference filters in the light beams limit the spectral transmittance to a well-defined wavelength range of about $40 \mathrm{~nm}$ before the light is detected by photomultipler tubes. A rotating backscatter shutter blade periodically blocks light emitted in the forward direction and thus allows measurement of the hemispheric backscatter signal. Specifications of TSI 3563 are given in Table 1.

\section{Truncation and angular illumination correction}

\subsection{Angular illumination functions}

Angular illumination functions for TSI 3563 were measured in a previous study (Müller et al., 2009) with a goniometer setup. For this study, illumination functions for the Aurora 
Table 1. Nephelometer specifications.

\begin{tabular}{|c|c|c|c|c|c|c|c|}
\hline \multirow[b]{2}{*}{ Type } & \multirow{2}{*}{$\begin{array}{l}\text { Light } \\
\text { source }\end{array}$} & \multirow{2}{*}{$\begin{array}{l}\text { Lambertian } \\
\text { diffuser }\end{array}$} & \multirow{2}{*}{$\begin{array}{l}\text { Wavelengths } \\
{[\mathrm{nm}]}\end{array}$} & \multirow{2}{*}{$\begin{array}{l}\text { Band-width } \\
\text { FWHM [nm] }\end{array}$} & \multirow[b]{2}{*}{ Backscatter } & \multicolumn{2}{|c|}{ Angular ranges } \\
\hline & & & & & & total scattering & backscattering \\
\hline TSI 3563 & $\begin{array}{l}\text { incandescent } \\
\text { halogen lamp }\end{array}$ & opal glass & $450,550,700$ & $\sim 40^{(1)}$ & continuously rotating & $7^{\circ}$ to $170^{\circ}$ & $90^{\circ}$ to $170^{\circ}$ \\
\hline Aurora 3000 & $\begin{array}{l}\text { three LEDs } \\
\text { for each } \\
\text { wavelength }\end{array}$ & opal glass & $450,525,635$ & $\begin{array}{l}<30^{(2)} \quad(450 \mathrm{~nm}) \\
<40^{(2)} \quad(525 \mathrm{~nm}) \\
<30^{(2)}(635 \mathrm{~nm})\end{array}$ & periodically moving & $10^{\circ}$ to $171^{\circ}$ & $90^{\circ}$ to $171^{\circ}$ \\
\hline
\end{tabular}

(1) Values are taken from Anderson et al. (1996). ${ }^{(2)}$ Values from specification sheets of LEDs.

3000 were measured using the same experimental setup with small modifications. We briefly describe here the principle of the angular intensity measurements.

The light source of the Aurora 3000 was removed and mounted on an optical bench in the centre of a computer controlled rotating arm. Relative emitted intensities were measured with a detection optics at the end the rotating arm at angles $0^{\circ}<\theta<180^{\circ}$ in steps of $5^{\circ}$. The detection optics consists of a lens which couples the light into an optical fibre and a photodiode attached to the other end of the fibre. Before measuring, it was ensured that the field of view of the detection optics was large enough to cover the whole surface of the opal glass of the light source. The overall uncertainty of the angular alignment was estimated to be less than one degree.

Measured angular intensity functions for the Aurora 3000 are shown in Fig. 2. Wavelengths are indicated by B $(450 \mathrm{~nm}), \mathrm{G}(525 \mathrm{~nm})$, and $\mathrm{R}(635 \mathrm{~nm})$, respectively, and total scattering and backscattering are indicated by ts and bs, respectively. Total scattering illumination functions were normalized to unity at a scattering angle of $90^{\circ}$ and the backward scattering illumination function was adjusted to match the value of the total scattering illumination function at $110^{\circ}$. Illumination functions for total scattering and for backscattering agree well among each other at large scattering angles $\left(\theta>110^{\circ}\right)$.

Illumination functions of TSI 3563 were measured independently and published in Anderson et al. (1996) and Müller et al. (2009). The illumination functions for total scattering agreed well for both studies. It also was found, that there is no difference between illumination functions for different nephelometer wavelengths.

Besides the non-Lambertian illumination, the angular truncation contributes to the overall angular sensitivity function. Truncation angles for the TSI 3563 were given in Anderson et al. (1996) and were reported to be $7^{\circ}$ and $170^{\circ}$. From technical drawings truncation angles for the Aurora 3000 were determined to be $10^{\circ}$ and $171^{\circ}$. To our knowledge there is currently no method for experimental verification of truncation angles.

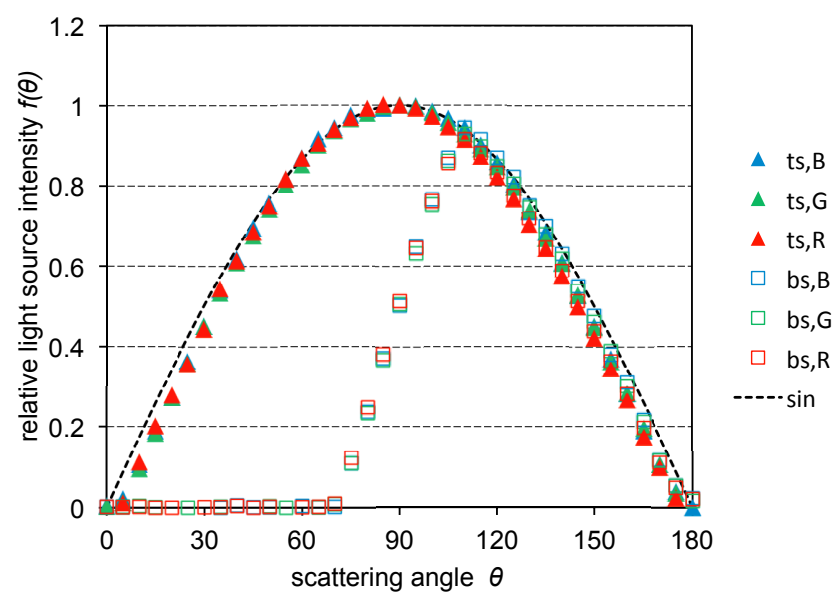

Fig. 2. Measured angular intensity functions for nephelometer model Aurora 3000 . The wavelengths are indicated by B $(450 \mathrm{~nm})$, $\mathrm{G}(525 \mathrm{~nm})$, and $\mathrm{R}(635 \mathrm{~nm})$, respectively. Total scattering and backscattering are indicated by ts and bs respectively. For comparison purposes, the total angular intensity functions have been normalized.

A parameterization was developed for total and backscattering sensitivity functions. The parameterizations are modifications of sine-functions with

$Z_{\mathrm{ts}}(\theta)=\left\{\begin{array}{cc}0 & 0^{\circ} \leq \theta \leq \alpha_{1} \\ \beta_{1} \times \sin (\theta)^{\beta_{2}} & \alpha_{1}<\theta<\alpha_{2} \\ 0 & \alpha_{2} \leq \theta \leq 180^{\circ}\end{array}\right.$

and

$Z_{\mathrm{bs}}(\theta)=\left\{\begin{array}{cc}0 & 0^{\circ} \leq \theta \leq \alpha_{1} \\ \max \left(0, \beta_{1} \times \sin (\theta)^{\beta_{2}} \times \min \left(1, \frac{\theta-\gamma_{1}}{\gamma_{2}}\right)\right) & \alpha_{1}<\theta<\alpha_{2} \\ 0 & \alpha_{2} \leq \theta \leq 180^{\circ}\end{array}\right.$

where $\alpha_{1}$ and $\alpha_{2}$ account for the upper and lower truncation and $\beta_{2}$ accounts for the decreased relative illumination at small and large angles. $\beta_{1}$ is a normalization factor, which cancels out in Eqs. (8) and (9). $\beta_{1}$ and $\beta_{2}$ are the same for total and backscattering. Theses parameters define the shape of the illumination curve for total scattering and that part of the backscatter illumination curve which is not blocked by 
Table 2. Parameters for truncation and non-Lambertian illumination correction functions. Correction functions are given for TSI model 3563 and Aurora 3000 nephelometers.

\begin{tabular}{lrrrrrr}
\hline Nephelometer & $\alpha_{1}$ & $\alpha_{2}$ & $\beta_{1}$ & $\beta_{2}$ & $\gamma_{1}$ & $\gamma_{2}$ \\
\hline TSI 3563(1) & $7^{\circ}$ & $170^{\circ}$ & 1.005 & 1.175 & 73.86 & 32.84 \\
Aurora 3000 & $10^{\circ}$ & $171^{\circ}$ & 1.01 & 1.190 & 70.25 & 39.99 \\
\hline
\end{tabular}

(1) Parameters were derived from sensitivity functions published in Müller et al. (2009).

the shutter. The shadowing of the backscatter shutter is accounted for by parameters $\gamma_{1}$ and $\gamma_{2}$. Parameters for TSI model 3563 and Aurora 3000 were derived from angular intensity functions shown in Müller et al. (2009) and Fig. 2, respectively. Parameters are given in Table 2. For comparison, Fig. 3 shows measured and parameterized illumination functions.

\subsection{Correction using measured size distributions}

Correction factors for angular sensitivity were calculated for the TSI 3563 and Aurora 3000 and are shown in Fig. 4 versus the volume median diameter $D_{\mathrm{Vm}}$ of a lognormal volume size distribution with geometric standard deviation 1.6. The refractive index was chosen to be 1.53-0.01i, which corresponds to a weakly absorbing aerosol. Corrections factors were calculated using Eqs. (8) and (9) with the parameterizations given in Eqs. (10) and (11) and the corresponding parameters given in Table 2. The wavelengths were $525 \mathrm{~nm}$ and $550 \mathrm{~nm}$ for the Aurora 3000 and TSI 3563, respectively. For total scattering, the correction factor increases for larger diameters and correction factors for the Aurora 3000 are higher than the corresponding values of the TSI 3563. Reasons for this are the different truncation angles in the forward scattering direction. The backscattering correction factors are smaller compared to total scattering with differences between the Aurora 3000 and TSI 3563 of less than $2 \%$.

\subsection{Correction using scattering Ångström exponents}

In Sect. 3.2, correction factors for angular illumination and truncation were derived from calculations based on Mie theory. Mie calculations require that particle number size distributions and complex refractive indices are known. Anderson and Ogren (1998), from now on denoted as AO98, found a dependency between the scattering Ångström exponent and the correction factor for total scattering, and presented a correction of the TSI 3563 nephelometer. The correction was given by

$C_{\mathrm{ts}}=a+b \cdot \alpha_{\mathrm{ts}}^{*}$,

where $\alpha_{\mathrm{ts}}^{*}$ is the scattering Ångström exponent derived from uncorrected nephelometer scattering. In AO98 it was pointed out that for backscattering no correlation between correction
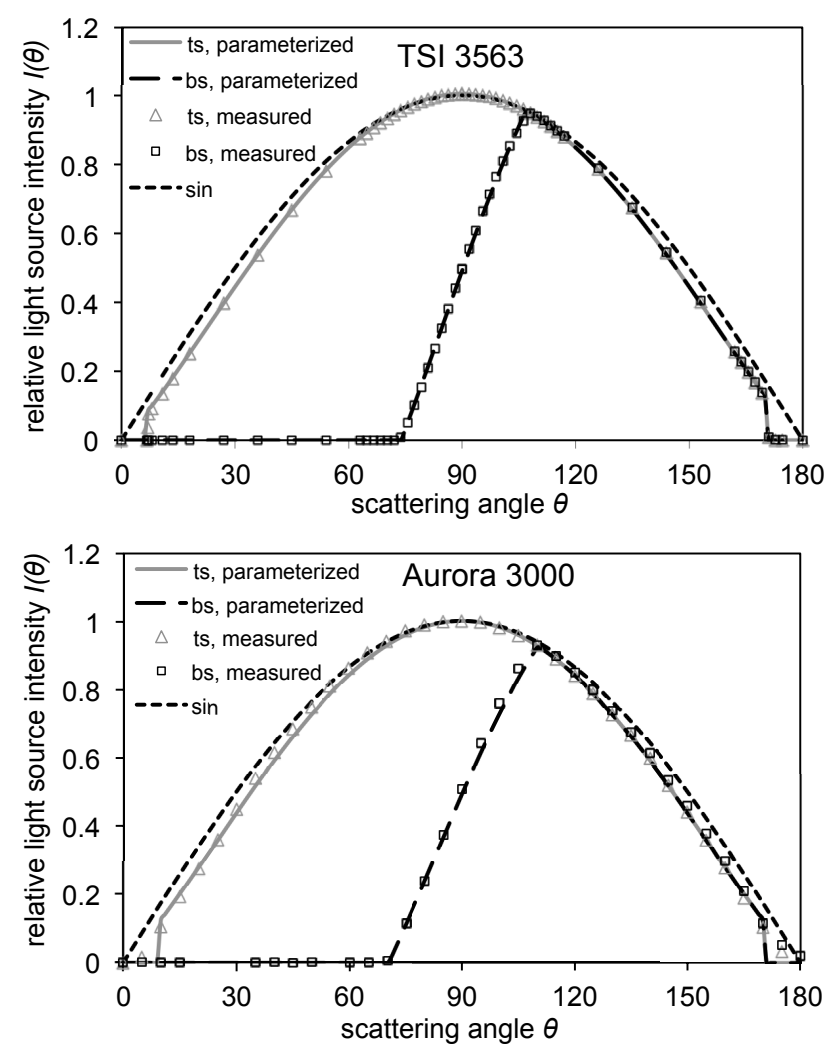

Fig. 3. Measured and parameterized angular intensity functions, $f(\theta)$, including forward and backward truncation for the TSI 3563 (upper panel, data are taken from Anderson et al., 1996) and Aurora 3000 (lower panel). For the Aurora 3000 the average of all three wavelengths is shown. For comparison purposes, the total scattering illumination function (ts) has been normalized to unity at $90^{\circ}$ scattering angle. The backward scattering illumination function (bs) was adjusted to match the total scattering function at $110^{\circ}$.

factors and scattering Ångström exponents was found. The parameters $a$ and $b$ were derived from Mie calculated true scattering and simulated nephelometer scattering for ranges of particle sizes and refractive indices. The calculations were limited to less absorbing particles with imaginary parts of the refractive index in the range $0 \leq \operatorname{im}(m) \leq 0.01$, and the real part of refractive index was in the range $1.40 \leq \mathrm{re}(\mathrm{m}) \leq 1.52$. Calculations were performed for bimodal lognormal size distributions with volume median diameters ranging from 0.2 to $0.4 \mu \mathrm{m}$ for the fine mode and 2.0 to $4.0 \mu \mathrm{m}$ for the coarse mode. Fine mode volume fractions were chosen between 0.1 and 0.9. Calculations were done twice with and without a sub- $\mu \mathrm{m}$ size cut. Calculations with sub- $\mu \mathrm{m}$ size cut were done for an aerodynamic diameter of $1 \mu \mathrm{m}$, which corresponds to a geometric diameter of $0.77 \mu \mathrm{m}$ for particles with a density of $1.7 \mathrm{~g} \mathrm{~cm}^{-3}$. We would like to annotate that nephelometers are mostly operated without any sub- $\mu \mathrm{m}$ cut. For correcting the scattering at the three wavelengths of the nephelometer, the parameterization uses scattering Ångström 

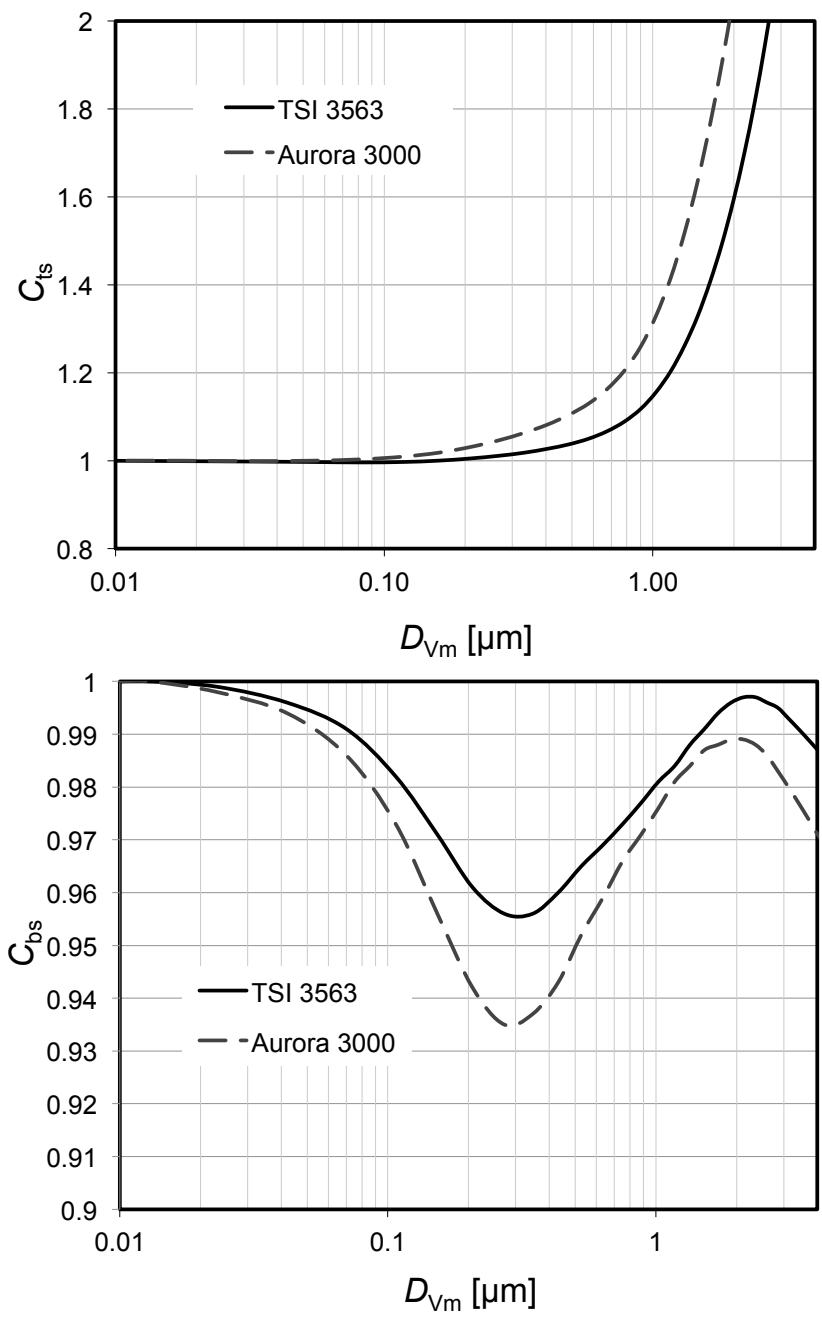

Fig. 4. Angular sensitivity correction factors for total scattering (upper panel) and backscattering (lower panel) versus the volume median diameter of a lognormal volume size distribution with geometric standard deviation 1.6. The refractive index of the aerosol was chosen to be 1.53-0.01i. The wavelength for calculating correction factors were 525 and $550 \mathrm{~nm}$ for Aurora 300 and TSI 3563, respectively.

exponents derived from different wavelength pairs. Wavelength pairs are $450 / 550$ for correction at $450 \mathrm{~nm}, 450 / 700$ for correction at $550 \mathrm{~nm}$, and $550 / 700$ for correction at $700 \mathrm{~nm}$, respectively.

In Bond et al. (2009) it is pointed out that the AO98 correction is accurate for a wide range of atmospheric aerosols. If the single scattering albedo is larger than 0.8 and the aerosol absorption does not have a large spectral dependence, the uncertainty of the correction is not expected to be larger than $2 \%$. For a large spectral dependence of the absorption, the uncertainty can be up to $3 \%$ at a single scattering albedo of 0.8 and larger for smaller single scattering albedos. It was found that changes in either the real or imaginary part of the refractive index result in a change of the scattering Ångström exponents. Even though, the truncation correction factor $C_{\mathrm{ts}}$ is rather non-sensitive to the refractive index. Thus, a correction as a function of the scattering Ångström exponent is limited in its use. In accordance with Bond et al. (2009), Massoli et al. (2009) reported high uncertainties of the AO98 correction for highly absorbing particles caused by the high real part of refractive indices of absorbing particles.

To be consistent with the frequently used AO98 correction for the TSI 3563 nephelometer, the same correction was derived for Aurora 3000. Mie calculations were done for the same range of parameters as given in AO98. Correction functions were also recalculated for TSI 3563 to check if the calculations are consistent with AO98. Results are given in Table 3. In agreement with Anderson and Ogren (1998) no correlation between backscattering correction factors and Ångström exponents was found. Therefore, Table $3 \mathrm{a}$ gives constant corrections factors and the corresponding uncertainties, defined as the half ranges of the correction factors. For total scattering, correction factors as a function of the Ångström exponent are given in Table $3 b$.

Figure 5 shows correction functions for no cut and sub$\mu \mathrm{m}$ cut conditions for total scattering. Recalculated correction factors for TSI 3563 agree well with the values given in AO98. Small differences between AO98 and this study might come from the choice of values for mode diameters and refractive indices within the given ranges. Additionally, the illumination function used in AO98 differs slightly from the parameterization of the illumination function used for this study. We would like to draw attention to the fact that the numbers of diameters and angles for numerically solving the integrals in Eq. (3) affects the result. For instance, the difference in truncation correction factors for angular resolutions of $1^{\circ}$ and $0.25^{\circ}$ can be up to $1.5 \%$. The results shown here were calculated with $0.25^{\circ}$. Differences in correction factors for TSI 3563 from this study and AO98 are smaller than $1 \%$.

From Fig. 5 we can see that the correction factors for Aurora 3000 are slightly higher than the correction for TSI 3563. For no-cut conditions and Ångström exponents between 0.5 and 2.0 the correction factors for Aurora 3000 are on average $5 \%$ higher than the correction factors for TSI 3563. With a sub- $\mu \mathrm{m}$ cut and Ångström exponents between 1.0 and 3.0, correction factors for Aurora 3000 are about $2 \%$ higher than for TSI 3563.

\section{Instrument characterization}

\subsection{Noise and detection limit}

Instrumental noise levels typically depend on the method of data filtering and averaging. The averaging in the TSI 3563 is done by a running-average which can be specified by the user. For the Aurora 3000, either a running-average filter or a digital Kalman filter can be selected. The Kalman filter 
Table 3a. Nephelometer correction factors for angular nonidealities. Wavelengths for TSI 3563 are $450 \mathrm{~nm}(\mathrm{~B}), 550 \mathrm{~nm}(\mathrm{G})$, and $700 \mathrm{~nm}$ (R), and wavelengths for Aurora 3000 are $450 \mathrm{~nm}(\mathrm{~B}), 525 \mathrm{~nm}(\mathrm{G})$, and $635 \mathrm{~nm}(\mathrm{R})$, respectively.

(a) Midpoint \pm half range of calculated correction factors for conditions with and without sub- $\mu \mathrm{m}$ cut

\begin{tabular}{|c|c|c|c|c|c|c|c|}
\hline & \multirow[b]{2}{*}{ wavelength } & \multicolumn{3}{|c|}{ total scatter } & \multicolumn{3}{|c|}{ backscatter } \\
\hline & & B & G & $\mathrm{R}$ & B & G & $\mathrm{R}$ \\
\hline \multirow[t]{2}{*}{ TSI 3563} & no cut & $1.30 \pm 0.25$ & $1.29 \pm 0.24$ & $1.26 \pm 0.21$ & $0.983 \pm 0.040$ & $0.984 \pm 0.041$ & $0.988 \pm 0.043$ \\
\hline & sub- $\mu \mathrm{m}$ & $1.086 \pm 0.040$ & $1.066 \pm 0.031$ & $1.045 \pm 0.021$ & $0.950 \pm 0.009$ & $0.944 \pm 0.012$ & $0.954 \pm 0.009$ \\
\hline \multirow[t]{2}{*}{ Aurora 3000} & no cut & $1.37 \pm 0.29$ & $1.38 \pm 0.31$ & $1.36 \pm 0.29$ & $0.963 \pm 0.040$ & $0.971 \pm 0.047$ & $0.968 \pm 0.043$ \\
\hline & sub- $\mu \mathrm{m}$ & $1.125 \pm 0.059$ & $1.103 \pm 0.046$ & $1.078 \pm 0.035$ & $0.932 \pm 0.012$ & $0.935 \pm 0.017$ & $0.935 \pm 0.014$ \\
\hline
\end{tabular}

Table 3b. Correction factors for total scatter as function of Ångström exponent: $C_{\mathrm{ts}}=a+b \times \alpha_{\mathrm{ts}}^{*}$. For correction of scattering coefficients for the blue (B) wavelength the Ångström exponent calculated from uncorrected scattering coefficients of blue and green (B/G) is used. At the wavelength $G$ and $R$ Ångström exponents at the wavelength pairs B/R and G/R are used, respectively.

\begin{tabular}{|c|c|c|c|c|c|c|c|}
\hline & wavelength & \multicolumn{2}{|c|}{ B } & \multicolumn{2}{|c|}{ G } & \multicolumn{2}{|c|}{$\mathrm{R}$} \\
\hline & Ångström exponents & \multicolumn{2}{|c|}{$\alpha_{\mathrm{ts}}^{*}(\mathrm{~B} / \mathrm{G})$} & \multicolumn{2}{|c|}{$\alpha_{\mathrm{ts}}^{*}(\mathrm{~B} / \mathrm{R})$} & \multicolumn{2}{|c|}{$\alpha_{\mathrm{ts}}^{*}(\mathrm{G} / \mathrm{R})$} \\
\hline & parameters & $a$ & $b$ & $a$ & $b$ & $a$ & $b$ \\
\hline \multirow[t]{2}{*}{ TSI 3563} & no cut & 1.345 & -0.146 & 1.319 & -0.129 & 1.279 & -0.105 \\
\hline & sub- $\mu \mathrm{m}$ & 1.148 & -0.041 & 1.137 & -0.040 & 1.109 & -0.033 \\
\hline \multirow[t]{2}{*}{ Aurora 3000} & no cut & 1.455 & -0.189 & 1.434 & -0.176 & 1.403 & -0.156 \\
\hline & sub- $\mu \mathrm{m}$ & 1.213 & -0.060 & 1.207 & -0.061 & 1.176 & -0.053 \\
\hline
\end{tabular}

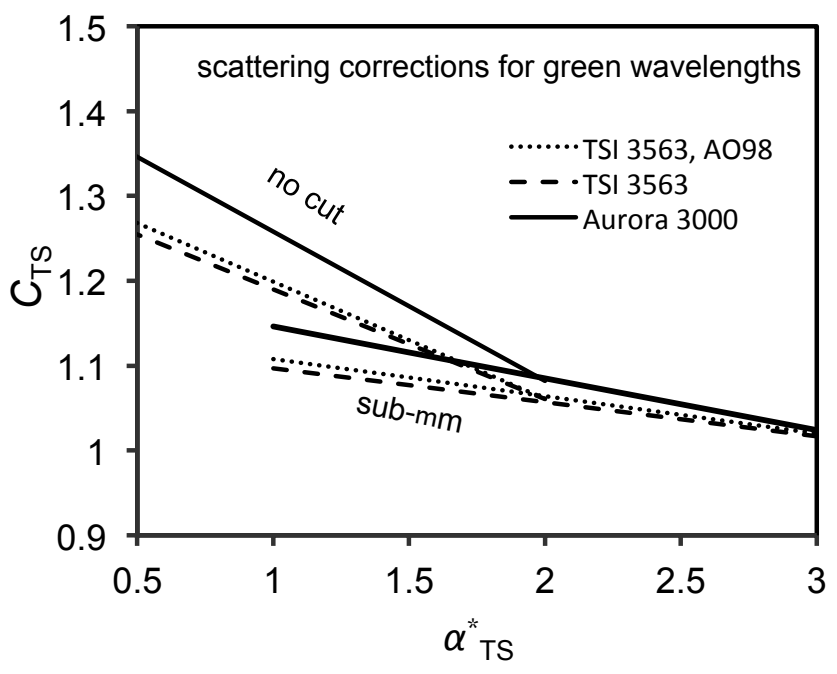

Fig. 5. Total scattering correction functions for the green wavelengths. Shown are two branches for no cut and sub- $\mu$ m cut.

provides a good compromise between response time and noise reduction. With the Kalman filter, the averaging time is variable and depends on the change rate of measured signals. For rapidly changing signals, the instrument responses quickly, while for steady signals, the effective averaging time increases. The measurements presented here were done with the Kalman filter, since this is the normal operation mode for ambient air monitoring. The use of different averaging and filtering methods does not allow a direct comparison of noise levels for both instruments.

Noise levels and detection limits were investigated in Anderson et al. (1996) for the TSI 3563. At low particle concentrations or short sampling times, the dominant source of uncertainties is random noise. The detection limits (signal to noise ratio of 2) for a five minute averaging time at wavelengths 450,550 , and $700 \mathrm{~nm}$ were given to be 0.29 , 0.11 , and $0.17 \mathrm{Mm}^{-1}$ for total scattering and $0.19,0.07$, and $0.11 \mathrm{Mm}^{-1}$ for backscattering, respectively. For the Aurora 3000, the noise was determined by sampling filtered air. Data were recorded minute by minute. The detection limits with Kalman filter at wavelengths 450,525 , and $635 \mathrm{~nm}$ were $0.11,0.14$, and $0.12 \mathrm{Mm}^{-1}$ for total scattering, and 0.12 , 0.11 , and $0.13 \mathrm{Mm}^{-1}$ for backscattering, respectively. An additional post averaging with five minute moving average changes the detection limit to $0.10,0.13$, and $0.11 \mathrm{Mm}^{-1}$ for total scattering and $0.09,0.11$, and $0.12 \mathrm{Mm}^{-1}$ for backscattering, respectively. The post averaging reduces the detection limit only a little, indicating that the effective averaging time during the noise tests is about five minutes or larger. 
Values for the detection limit are in agreement with the detection limit of $<0.3 \mathrm{Mm}^{-1}$ given in the user manual for all wavelengths. Results indicate that the noise of TSI 3563 and Aurora 3000 might be similar. Because of the different filter and averaging methods, a better comparison of noise and detection limit was not possible.

The noise for backscattering is similar to that for total scattering. However, the signal to noise ratio for backscattering is worse compared to total scattering, since the backscattering signal is about one order of magnitude smaller than the total scattering signal for ambient air. Drifts of background signals caused by wall scattering and detector offset are compensated by continuous zero measurements during normal operation and during calibration. A drift between two zero measurements can cause a bias, which is worse for backscattering than for total scattering. Thus the backscatter channels are more sensitive to non stable operation conditions.

\subsection{Calibration}

The accuracy of gas calibrations was investigated in Heintzenberg et al. (2006) with many nephelometers. Span gas $\left(\mathrm{CO}_{2}\right)$ checks with 15 instruments of type TSI 3563 were performed and showed total scattering average errors ( $90 \%$ confidence) of $3 \%$. For backscattering, errors less than $5 \%$ were found during a calibration and intercomparison workshop (Müller et al., 2009) but not published before. The uncertainty of calibration for the Aurora 3000 is given in the user manual to be $2.5 \%$ (Ecotech, 2009). Span gas checks by a series of calibrations with one or multiple Aurora 3000 nephelometers in parallel have not been performed to date. Thus, an independent test of calibration uncertainties by means of span gas is not available.

\section{Instrument comparison}

\subsection{Laboratory comparison: ammonium sulphate}

\subsubsection{Setup}

A solution of ammonium sulfate was nebulized as a test aerosol with known composition and refractive index. The aerosol was dried by diluting it with dry particle free air to relative humidities below $10 \%$. The aerosol was fed into a 15 litre mixing tank upstream of the Aurora 3000 and TSI 3563 nephelometers. Particle number size spectra were measured with an optical particle size spectrometer (UHSAS, Droplet Measurement Technologies, Boulder, CO, USA) in the size range 0.06 to $1.0 \mu \mathrm{m}$. Particle size classification with optical spectrometers depends on the particles refractive index. Since refractive indices of ammonium sulfate and polystyrene latex, which was used for calibration of UHSAS, are known, size spectra were corrected to a volume equivalent particle diameter. Particle number size distributions were used to calculate correction factors for angular sensitivity functions. The volume median diameter was on average $0.24 \mu \mathrm{m}$ with lower (10th percentile) and upper (90th percentile) diameters of $0.20 \mu \mathrm{m}$ and $0.68 \mu \mathrm{m}$, respectively.

\subsubsection{Instrument comparison}

For comparison, scattering and backscattering coefficients measured by TSI 3563 were adjusted to wavelengths 450 , 525 and $635 \mathrm{~nm}$ using the Ångström exponents defined by

$\alpha_{\mathrm{ts}, \lambda_{1}, \lambda_{2}}=-\ln \left(\sigma_{\mathrm{ts}, \sigma_{1} / \lambda_{2}}\right) / \ln \left(\lambda_{1} / \lambda_{2}\right)$.

and

$\alpha_{\mathrm{bs}, \lambda_{1}, \lambda_{2}}=-\ln \left(\sigma_{\mathrm{bs}, \lambda_{1}} / \sigma_{\mathrm{bs}, \lambda_{2}}\right) / \ln \left(\lambda_{1} / \lambda_{2}\right)$.

Ångström exponents $\alpha_{\mathrm{ts}, \lambda_{1}, \lambda_{2}}$ and $\alpha_{\mathrm{bs}, \lambda_{1}, \lambda_{2}}$ are calculated from scattering coefficients at wavelengths $\lambda_{1}$ and $\lambda_{2}$. Once the Ångström exponents are calculated from scattering at two nephelometer wavelength, Eqs. (12) and (13) can be used to adjust scattering and backscattering coefficients to any other wavelength $\lambda_{\mathrm{x}}$ by $\sigma_{\lambda_{\mathrm{x}}}=\sigma_{\lambda_{1,2}}\left(\lambda_{1,2} / \lambda_{\mathrm{x}}\right)^{\alpha_{1,2}}$, where $\lambda_{1,2}$ is one of the nephelometer wavelengths and $\sigma_{\lambda_{1,2}}$ the corresponding scattering coefficient. Angular sensitivities were corrected using Mie-calculation with measured size distributions from the optical particle counter and the refractive index of ammonium sulfate $(m=1.53-0 i$ at $550 \mathrm{~nm})$.

Scatter-plots of total and backscattering are shown in Fig. 6. Results of a linear regression analysis for uncorrected as well as corrected scattering coefficients are summarized in Table 4a. Corrected total scattering coefficients measured by the Ecotech Aurora 3000 and TSI 3563 differ between $2 \%$ and $5 \%$. Differences in backscattering are between $1 \%$ and $11 \%$. Differences for total scattering are in agreement within the uncertainties of calibration. The highest deviation occurs for the red channels in backscattering mode and cannot be explained by calibration uncertainties. Both nephelometers have the lowest signal to noise ratios for this channel. The intercepts of all linear regressions are small and close to the sum of detection limits of both nephelometers.

A statistical analysis of Ångström exponents is given in Table 5. Ångström exponents for total scattering differ by $5 \%$ and $1 \%$ for wavelength pairs $450 / 525$ and 525/635, respectively. Differences in backscattering are higher and amount $11 \%$ and $17 \%$ for the same wavelength pairs. It cannot clearly be explained why differences in backscattering are higher than for total scattering, and why the relationships change with wavelength. We suspect that the low signal for backscattering, especially in the red wavelength, causes higher uncertainties since the backscattering is more sensitive to non stable operation conditions (c.f. Sect. 4.1). 

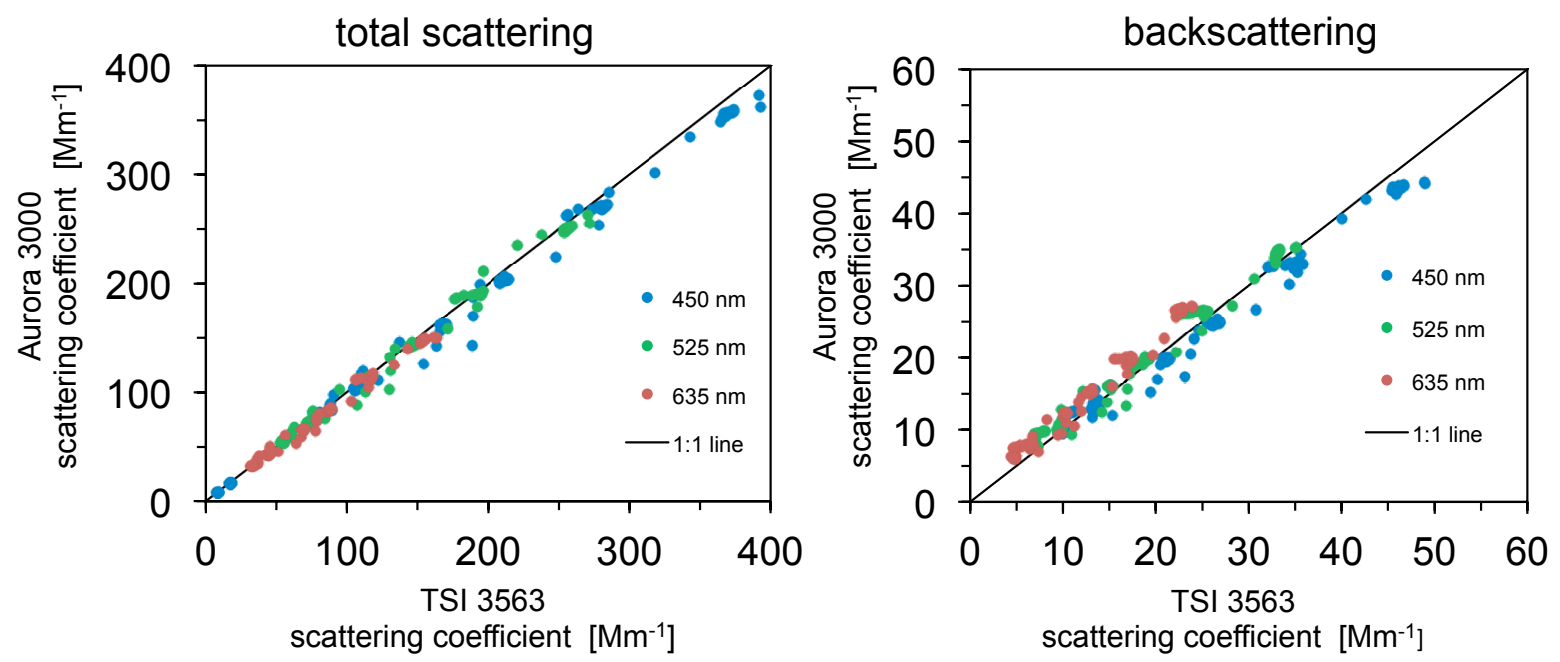

Fig. 6. Results from experiments with ammonium sulphate. Correlation between total scattering coefficients measured with the TSI 3563 and Aurora 3000, respectively. The left panel shows the correlation for total scattering coefficients including truncation correction. Wavelengths for TSI 3563 were adjusted to 450, 525, and $635 \mathrm{~nm}$. The right panel is similar to the left panel but for backscattering coefficients.

Table 4. Results of regression analysis $\left(\sigma_{\text {Aurora3000 }}=m\right.$ $\sigma_{\mathrm{TSI} 3563}+b$ and the coefficient of determination $R^{2}$ ) of the Aurora 3000 and TSI 3563 for total scattering (ts) and backscattering (bs). The intercept $b$ is given in units of $\mathrm{Mm}^{-1}$. Directly measured values as well as angular sensitivity corrected values are shown. Wavelengths are $B=450 \mathrm{~nm}, \mathrm{G}=525 \mathrm{~nm}$, and $\mathrm{R}=635 \mathrm{~nm}$. Values are given for (a) ammonium sulphate and (b) ambient air.

(a) Ammonium sulphate

\begin{tabular}{lrrrrrr}
\hline & ts, B & ts, G & ts, R & bs, B & bs, G & bs, R \\
\hline & \multicolumn{5}{c}{ without angular sensitivity correction } \\
\cline { 2 - 7 } slope $(m)$ & 0.94 & 0.96 & 0.94 & 0.95 & 1.04 & 1.13 \\
intercept $(b)$ & 0.51 & 0.64 & 0.94 & 0.46 & 0.61 & 0.88 \\
$R^{2}$ & 0.998 & 0.995 & 0.996 & 0.993 & 0.991 & 0.988 \\
\hline \multicolumn{6}{c}{ with angular sensitivity correction } \\
slope $(m)$ & 0.96 & 0.98 & 0.95 & 0.93 & 1.01 & 1.11 \\
intercept $(b)$ & 0.56 & 0.68 & 0.97 & 0.43 & 0.57 & 0.81 \\
$R^{2}$ & 0.998 & 0.995 & 0.996 & 0.993 & 0.991 & 0.998 \\
\hline
\end{tabular}

\begin{tabular}{lrrrrrr}
\hline (b) Ambient air & & & \\
& ts, B & ts, G & ts, R & bs, B & bs, G & bs, R \\
\hline \multirow{7}{c}{ without angular sensitivity correction } \\
\cline { 2 - 7 } slope $(m)$ & 0.96 & 0.98 & 0.93 & 1.04 & 1.05 & 0.98 \\
intercept $(b)$ & 1.38 & 1.08 & 0.45 & -0.07 & -0.14 & 0.06 \\
$R^{2}$ & 0.997 & 0.997 & 0.997 & 0.994 & 0.994 & 0.995 \\
\hline & \multicolumn{7}{c}{ with angular sensitivity correction } \\
\cline { 2 - 7 } slope $(m)$ & 0.99 & 1.01 & 0.95 & 1.01 & 1.03 & 0.96 \\
intercept $(b)$ & 1.04 & 0.86 & 0.26 & -0.11 & -0.16 & -0.03 \\
$R^{2}$ & 0.998 & 0.997 & 0.997 & 0.993 & 0.994 & 0.994 \\
\hline
\end{tabular}

\subsection{Ambient air}

\subsubsection{Setup, aerosol characterization, and truncation correction}

An ambient air intercomparison was performed for a period of 46 days in December 2009 and January 2010. The aerosol was fed through an aerosol inlet and custom-made Nafion ${ }^{\mathrm{TM}}$ membrane diffusion dryers into a mixing chamber, which ensures equal aerosol concentrations at all output ports of the chamber. The aerosol was dried to a relative humidity of less than $25 \%$. Transport losses through the inlet, Nafion dryer, mixing chamber and aerosol transport lines into the chamber are not specified. Transport losses from the chamber to the instruments were estimated to be small and did not affect the instrument comparison.

Particle number size distributions were measured with a custom-made SMPS (Scanning mobility particle sizer, Wang and Flagan, 1989) for mobility diameters $d_{m}$ between 30 and $800 \mathrm{~nm}$. Aerodynamic size spectra were measured with an APS (Aerodynamic Particle Sizer, model 3321, TSI Inc., St. Paul, MN) from aerodynamic diameters $d_{\text {aer }}$ between 542 $\mathrm{nm}$ and $19.81 \mu \mathrm{m}$. The relations between mobility diameter, aerodynamic diameter and diameter of volume equivalent spheres $d_{\mathrm{veq}}$ are given by $d_{\mathrm{veq}}=d_{m} / \chi$ for SMPS and $d_{\mathrm{veq}}=d_{\mathrm{aer}} / \sqrt{\rho / \chi}$ for APS, where $\rho$ and $\chi$ are the particle density in units of $\mathrm{g} \mathrm{cm}^{-3}$ and the dynamic shape factor, respectively (e.g. DeCarlo et al., 2004). Because no information about the particle shape and density were available, the particles were considered to be spherical with a dynamic shape factor of unity and the particle density was assumed to be $1.7 \mathrm{~g} \mathrm{~cm}^{-3}$, which roughly corresponds to ammonium sulfate. Size spectra from SMPS and APS were combined 
Table 5. Ångström exponents for two wavelength pairs and angular sensitivity corrected total and backscattering, respectively. Given are the median and 10th and 90th percentiles for two wavelength pairs and total and backscattering, respectively.

\begin{tabular}{|c|c|c|c|c|c|c|c|c|}
\hline \multirow{4}{*}{$\begin{array}{l}\text { wavelengths pair }[\mathrm{nm}] \\
\text { median }\end{array}$} & \multicolumn{4}{|c|}{ TSI 3563} & \multicolumn{4}{|c|}{ Aurora 3000} \\
\hline & \multicolumn{2}{|c|}{ total scattering } & \multicolumn{2}{|c|}{ backscattering } & \multicolumn{2}{|c|}{ total scattering } & \multicolumn{2}{|c|}{ backscattering } \\
\hline & $450 / 525$ & $525 / 635$ & $450 / 525$ & $525 / 635$ & $450 / 525$ & $525 / 635$ & $450 / 525$ & $525 / 635$ \\
\hline & 2.41 & 2.78 & 1.64 & 1.59 & 2.28 & 2.79 & 1.48 & 1.36 \\
\hline 10th percentile & 2.37 & 2.74 & 1.50 & 1.50 & 2.24 & 2.74 & 1.35 & 1.17 \\
\hline 90th percentile & 2.46 & 2.83 & 1.74 & 1.73 & 2.32 & 2.84 & 1.62 & 1.52 \\
\hline
\end{tabular}

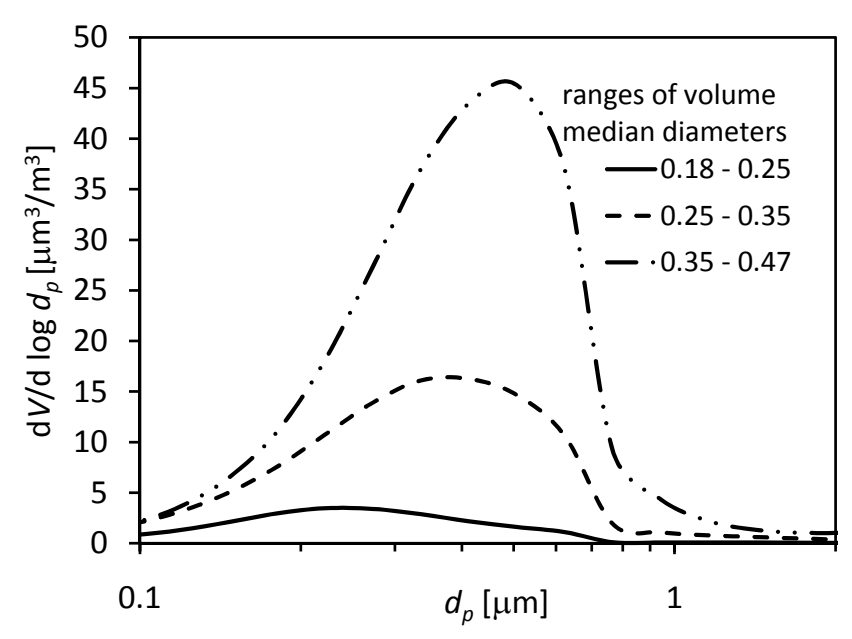

Fig. 7. Average particle volume size distributions for ambient air classified for three ranges of volume median diameters.

to a particle number size distribution, which was used to calculate volume size distributions $\mathrm{d} V\left(d_{\mathrm{p}}\right) / \mathrm{d} \log d_{\mathrm{p}}$ and volume median diameters $D_{V} \mathrm{~m}$. For an additional aerosol characterization, the particle absorption coefficient was measured with a MAAP (Multi angle absorption photometer, Thermo Fisher Scientific Inc.,Waltham, USA)

Correction of the truncation error can be done either by Mie-calculations of by the Angström parameterization, which can be used for less absorbing particles with imaginary parts of the refractive index smaller than 0.01 . In the following section, we present an estimation of the imaginary part of refractive index.

Measured average volume size distributions for three ranges of volume median diameters are shown in Fig. 7. The particle volume concentration ranges between 1.5 and $60 \mu \mathrm{m}^{3} \mathrm{~m}^{-3}$ and the range of volume median diameter is between 0.2 and about $0.48 \mu \mathrm{m}$. Ambient air particles are a mixture of different compounds as easily can be seen by the volume size spectra shown in Fig. 7. An external mixing state means that individual particles consist of a single compound. In internally mixed aerosol, several compounds can be found in individual particles. The mixing is important, when considering absorption, whereas the scattering coefficient in contrast is less sensitive to the mixing state. Since we have no information about the mixing state, we assume an internally mixed aerosol with a size-independent complex refractive index. This assumption might be supported by backward trajectories, which indicate that the air mass during the comparison measurements was an aged continental aerosol.

The complex refractive index $m=n$-i $k$ was calculated by an iterative algorithm. Differences between simulated and measured scattering and absorption were minimized by optimizing the imaginary part of the refractive index until the minimization function $\zeta(k)$, defined by defined by

$\zeta(k)=\sqrt{\left(\frac{\sigma_{\mathrm{ts}, 525 \mathrm{~nm}}^{\mathrm{neph}}-\sigma_{\mathrm{ts}, 525 \mathrm{~nm}}^{\mathrm{Mie}}(k)}{\sigma_{\mathrm{ts}, 525 \mathrm{~nm}}^{\mathrm{neph}}}\right)^{2}+\left(\frac{\sigma_{\mathrm{abs}, 637 \mathrm{~nm}}^{\mathrm{MAAP}}-\sigma_{\mathrm{abs}, 637 \mathrm{~nm}}^{\mathrm{Mie}}(k)}{\sigma_{\mathrm{abs}, 637 \mathrm{~nm}}^{\mathrm{MAAP}}}\right)^{2}}$

has its minimal value. The measured and calculated values are indicated by upper indices "neph" and "Mie" respectively. The real part of the refractive index was chosen to be $n=1.53$, a typical value for low absorbing ambient aerosol mainly consisting of ammonium sulphate (Wex et al., 2002). Minimization was done using the nephelometer scattering coefficients at $525 \mathrm{~nm}$ measured with the $\mathrm{Au}$ rora 3000 and the absorption coefficients measured at $637 \mathrm{~nm}$ with the MAAP. The median value of the time series of the imaginary part of refractive index was 0.048 and the 95th and 5 th percentiles were 0.082 and 0.023 , respectively. Because of the occasionally high imaginary parts of refractive index, truncation correction factors were not calculated using the Ångström parameterization. Instead, scattering coefficients were corrected using Mie-theory with the time series of particle numbers size distributions and estimated refractive indices.

\subsubsection{Nephelometer comparison}

Total scattering and backscattering coefficients were compared using the measured data of the Aurora 3000 and TSI 3563. Figure 8 shows angular sensitivity corrected scattering and backscattering at wavelengths $525 \mathrm{~nm}$ for both nephelometer models. There is an excellent agreement between 
both instruments with a coefficient of determination $\left(R^{2}\right)$ higher than 0.99 for both total scattering and backscattering. Deviations in the slope are small with about $1 \%$ and $3 \%$ for total scattering and backscattering, respectively. Linear fit parameters for all three wavelengths and for uncorrected as well as corrected values are summarized in Table 4b. In all cases the coefficients of determination are better than 0.99. For uncorrected scattering, the slopes averaged for all three wavelengths are 0.96 and 1.02 for total scattering and backscattering, respectively. Average slopes after applying angular sensitivity correction corrections are 0.98 and 1.00 . Differences in the slopes of the blue and green channels are less than $2 \%$. Differences in the red wavelength compared to the other wavelengths are about $6 \%$. Higher deviations in the red channels cannot be explained. Deviations in the slope for the blue and green channels are less than the uncertainties of calibration, showing that the instruments agree well within the specified uncertainties.

Measurements of Ångström exponents are often applied for aerosol characterization. Changes of Ångström exponents indicate changes of the volume median diameter of the particle population, whereas a higher Ångström exponent indicates smaller volume median diameters. We compared Ångström exponents derived from the Aurora 3000 and TSI 3563. Ratios of Ångström exponents $\left(\alpha_{\mathrm{R}}\right)$, defined by Ångström exponents at 450 and $525 \mathrm{~nm}$ from the Aurora 3000 divided by Ångström exponents from the TSI 3563 at the same wavelengths, were investigated. Fig. 9 shows ratios of Ångström exponents versus the volume median diameter for total scattering and backscattering as well as for uncorrected and angular sensitivity corrected values. When considering total scattering, the slope of the regression line is significantly better for angular sensitivity corrected values. For backscattering, the ratios of Angström exponents are similar for the cases with and without angular sensitivity correction. The majority of volume median diameters were between 0.2 and $0.4 \mu \mathrm{m}$. In this range, the Ångström exponents with angular sensitivity correction differ between $3 \%$ and $11 \%$ for total scattering, with the TSI 3563 reading the higher values. For backscattering, the Aurora is higher by $8 \%$ for the smaller volume median diameters and lower by $6 \%$ for the larger volume median diameters. The noise (standard deviation) of the ratios is $6 \%$ for total scattering. The noise for backscattering ratios is much higher with $28 \%$, which can be explained by the much smaller signal to noise ratio for the backscatter signal compared to total scattering.

In all cases, there is the tendency that the ratio becomes smaller for larger volume median diameters. For corrected scattering and backscattering, this effect is less than for uncorrected scattering. For ideals nephelometers and corrections, the ratio should be independent on the size. It is unlikely that uncertainties of the gas calibration are responsible for the tendency. Faulty calibration factors at one or two wavelengths can cause a constant bias in the Ångström exponents, but they cannot explain the observed size dependence a) corrected scattering coefficients $\left[\mathrm{Mm}^{-1}\right]$

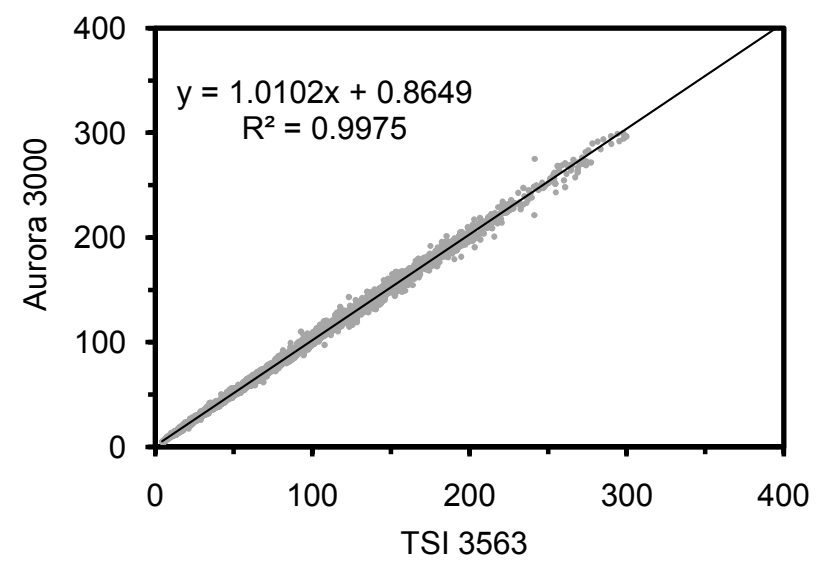

b) corrected backscattering coefficients $\left[\mathrm{Mm}^{-1}\right]$

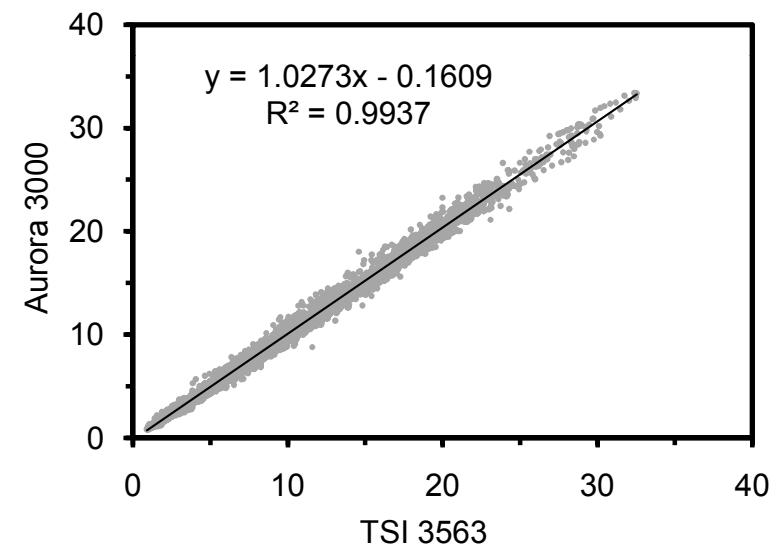

Fig. 8. Results from experiments with ambient air. Panel (a) Correlation between total scattering coefficients measured with the TSI model 3563 and Aurora 3000, respectively. Panel (b) shows the correlation for backscattering coefficients. Total scattering and backscattering at $525 \mathrm{~nm}$ were corrected for angular sensitivities. TSI 3525 scattering coefficients ware adjusted to wavelength $525 \mathrm{~nm}$ using an interpolation based on the Ångström exponents.

in the ratio of Ångström exponents. Possibly, truncation angles were not determined correctly for either one or both of the nephelometers, or truncation angles were not determined in exactly the same way. Unfortunately, there is no experimental method for measuring the nephelometer angular sensitivity including angular truncation, illumination function, and the cell dimension in a single setup. This deficiency prevents a better characterization of nephelometers.

\section{Summary}

We presented the performance of the new Ecotech Aurora 3000 integrating nephelometer. The Aurora 3000 is a threewavelength total and backscatter integrating nephelometer. 
a) total scattering without corrections

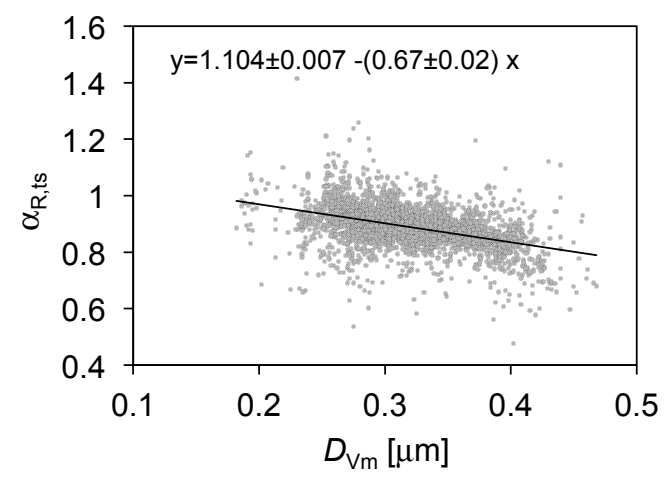

c) backscattering without corrections

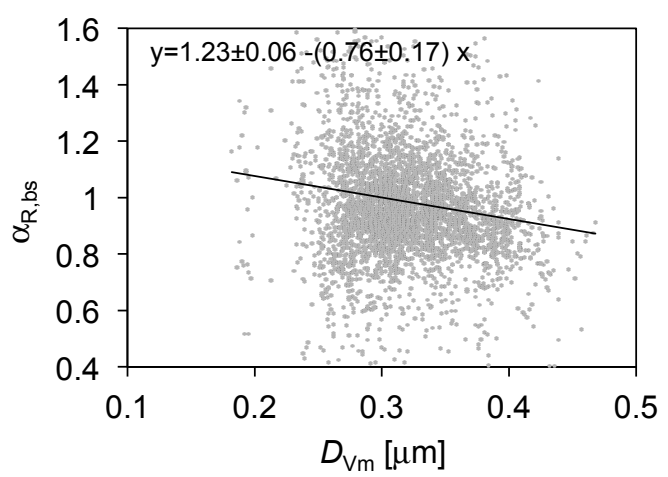

b) total scattering with corrections

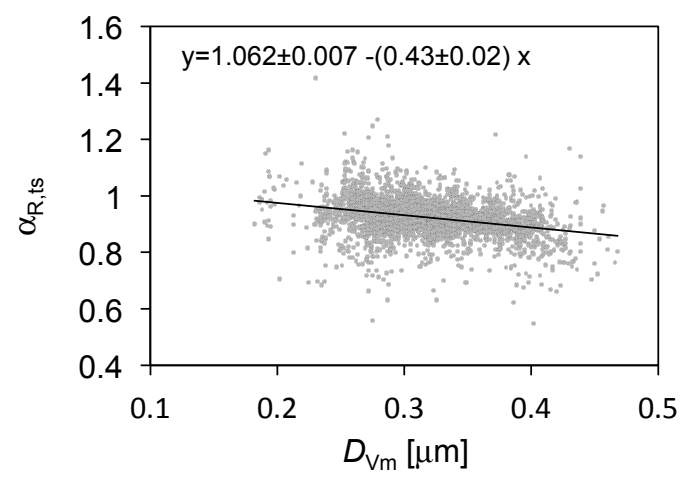

d) backscattering with corrections

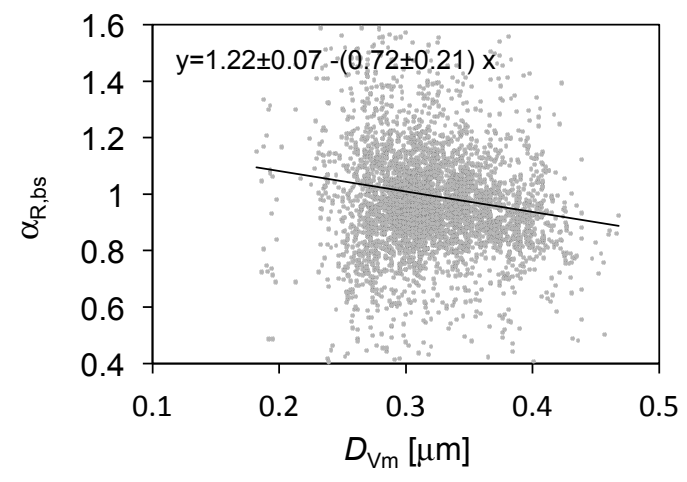

Fig. 9. Results from ambient air experiments. Ratios of Ångström exponents $\left(\alpha_{R}\right)$ at wavelength 450 and 525 nm, defined by Ångström exponents from the Aurora 3000 divided by Ångström exponents from the TSI 3563. Panel (a) and (b) show ratios for uncorrected and sensitivity corrected total scattering. Ratios for uncorrected and sensitivity corrected backscattering are shown in panels (c) and (d).

The light source consists of three LEDs per wavelength. An opal glass diffuser provides a nearly Lambertian angular illumination function, which is cut by the cell geometry at truncation angles of $10^{\circ}$ and $171^{\circ}$. A periodically moving shutter allows measurement of hemispheric backscattering. An intercomparison of the Ecotech Aurora 3000 and the TSI 3563 three-wavelength total scatter and backscatter integrating nephelometers was performed in this investigation. To correct systematic uncertainties due to angular truncation and non-Lambertian illumination, truncation angles were obtained from technical drawings and angular illumination functions of the Ecotech Aurora 3000 were measured and compared to the TSI model 3563 nephelometer. Currently, there is no method to verify truncation angles. Even the uncertainty of truncation angles is unknown. For better understanding of nephelometer corrections, it is recommended that effective truncation angles are determined experimentally. The TSI model 3563 was previously characterized in detail by Anderson et al. (1996) and Müller et al. (2009). Parameterizations of angular sensitivities for total scattering and backscattering were derived for both models of nephelometers along with correction factors as a function of Angstrom exponent.
The Ecotech Aurora 3000 and TSI 3563 integrating nephelometers were compared using ammonium sulphate and ambient air aerosols. Scattering and backscattering coefficients at wavelengths 450 and $525 \mathrm{~nm}$ agree well within the calibration uncertainties. Calibration uncertainties are less than $3 \%$ and $5 \%$ for total and backscattering, respectively. Maximum differences in total scattering are $4 \%(450 \mathrm{~nm}), 2 \%$ $(525 \mathrm{~nm})$ and $5 \%(635 \mathrm{~nm})$ for experiments with ambient air and laboratory generated white particles. For backscattering, the differences are higher and amount $7 \%(450 \mathrm{~nm})$, $3 \%(525 \mathrm{~nm})$ and $11 \%(635 \mathrm{~nm})$. Ångström exponents for a large range of volume median diameters were investigated during an ambient air experiment. Ångström exponents derived at the wavelength pair 450 and $525 \mathrm{~nm}$ agree well and differ by less than $5 \%$ for particle populations with volume median diameter less than $0.3 \mu \mathrm{m}$. Ratios of Ångström exponents for both nephelometer models show a slight dependence on volume median diameters. A sound explanation of this size effect is missing. However, values of scattering and backscattering coefficients of both nephelometers agree well. 
Acknowledgements. The IfT-authors would like to state that this work was done without financial support of the Ecotech Company. Results were objectively assessed and are uninfluenced by personal opinions.

Edited by: B. Mayer

\section{References}

Anderson, T. L. and Ogren, J. A.: Determining aerosol radiative properties using the TSI 3563 integrating nephelometer, Aerosol Sci. Tech., 29, 57-69, 1998.

Anderson, T. L., Covert, D. S., Marshall, S. F., Laucks, M. L., Charlson, R. J., Waggoner, A. P., Ogren, J. A., Caldow, R., Holm, R. L., Quant, F. R., Sem, G. J., Wiedensohler, A., Ahlquist, N. A., and Bates, T. S.: Performance characteristics of a high-sensitivity, three-wavelength, total scatter/backscatter nephelometer, J. Atmos. Oceanic Technol., 13, 967-986, 1996.

Andrews, E., Sheridan, P. J., Fiebig, M., McComiskey, A., Ogren, J. A., Arnott, P., Covert, D., Elleman, R., Gasparini, R., Collins, D., Jonsson, H., Schmid, B., and Wang, J.: Comparison of methods for deriving aerosol asymmetry parameter, J. Geophys. Res.Atmos., 111, D05S04, doi:10.1029/2004JD005734, 2006.

Beuttell, R. G. and Brewer, A. W.: Instruments for the measurement of the visual range, J. Sci. Instr. Phys. Ind., 26, 357-359, 1949.

Bohren, C. F. and Huffman D. R., Absorption and Scattering of Light by Small Particles, Wiley, New York, 1983.

Bond, T. C., Covert, D. S., and Müller, T., Truncation and AngularScattering Corrections for Absorbing Aerosol in the TSI 3563 Nephelometer, Aerosol Sci. Tech., 43, 866-871, 2009.

Buchholz, A.: Rayleigh scattering calculations for the terrestrial atmosphere, Appl. Optics, 34, 2765-2773, 1995.

Charlson, R. J., Porch, W. M., Waggoner, A. P., and Ahlquist, N. C.: Background aerosol light-scattering characteristics - nephelometric observations at Mauna-Loa-observatory compared with results at other remote locations, Tellus, 26, 345-360, 1974.

DeCarlo, P., Slowik, J., Worsnop, D., Davidovits, P., and Jimenez, J.: Particle Morphology and Density Characterization by Combined Mobility and Aerodynamic Diameter Measurements, Part 1: Theory, Aerosol Sci. Tech., 38, 1185-1205, 2004.
Ecotech, Aurora 3000 User manual 1.3, November 2009.

Heintzenberg, J.: The angular calibration of the total scatter/backscatter nephelometer, consequences and application, Staub-Reinhalt. Luft, 38, 62-63, 1978.

Heintzenberg, J. and Charlson, R. J.: Design and applications of the integrating nephelometer: A review, J. Atmos. Oceanic Technol., 13, 987-1000, 1996.

Heintzenberg, J., Wiedensohler, A., Tuch, T. M., Covert, D. S., Sheridan, P., Ogren, J. A., Gras, J., Nessler, R., Kleefeld, C., Kalivitis, N., Aaltonen, V., Wilhelm, R. T., and Havlicek, M.: Intercomparisons and aerosol calibrations of 12 commercial integrating nephelometers of three manufacturers, J. Atmos. Oceanic Technol., 23, 902-914, 2006.

Marshall, S. F., Covert, D. S., and Charlson, R. J.: Relationship between asymmetry parameter and hemispheric backscatter ratio - implications for climate forcing by aerosols, Appl. Optics, 34, 6306-6311, 1995.

Massoli, P., Murphy, D. M., Lack, D. A., Baynard, T., Brock, C. A., and Lovejoy, E. R.: Uncertainty in Light Scattering Measurements by TSI Nephelometer: Results from Laboratory Studies and Implications for Ambient Measurements, Aerosol Sci. Tech. 43, 1064-1074, 2009.

Moosmüller, H. and Arnott, W. P.: Angular truncation errors in integrating nephelometry, Rev. Sci. Instrum., 74, 3492-3501, 2003.

Müller, T., Nowak, A., Wiedensohler, A., Sheridan, P., Laborde, M., Covert, D. S., Marinoni, A., Imre, K., Henzing, B., Roger, J. C., dos Santos, S. M., Wilhelm, R., Wang, Y. Q., and de Leeuw, G.: Angular Illumination and Truncation of Three Different Integrating Nephelometers: Implications for Empirical, Size-Based Corrections, Aerosol Sci. Tech., 43, 581-586, 2009.

Wang, S. C. and Flagan, R. C.: Scanning electrical mobility spectrometer, J. Aerosol Sci., 20, 1485-1488, 1989.

Wex, H., Neusüß, C., Wendisch, M., Stratmann, F., Koziar, C., Keil, A., Wiedensohler, A., and Ebert, M: Particle scattering, backscattering, and absorption coefficients: An in situ closure and sensitivity study, J. Geophys. Res.-Atmos. 107(D21), 8122, doi:10.1029/2000JD000234, 2002. 